\title{
The square mean of Dirichlet series associated with cusp forms
}

\author{
Journal Article
}

Author(s):

Good, Anton

Publication date:

1982

Permanent link:

https://doi.org/10.3929/ethz-b-000422879

Rights / license:

In Copyright - Non-Commercial Use Permitted

Originally published in:

Mathematika 29(2), https://doi.org/10.1112/S0025579300012377 


\section{THE SQUARE MEAN OF DIRICHLET SERIES ASSOCIATED WITH CUSP FORMS}

\section{ANTON GOOD}

§1. Introduction. Let

$$
F(z)=\sum_{l=1}^{\infty} a_{l} e^{2 \pi i l z}, \quad \operatorname{Im} z>0,
$$

be a cusp form of even integral weight $k>2$ for the full modular group. Then the Dirichlet series

$$
L_{F}(s)=\sum_{l=1}^{\infty} a_{l} l^{-s}, \quad s=\sigma+i t
$$

is absolutely convergent for $\sigma>\frac{1}{2}(k+1)$. Hecke showed that $L_{F}$ is an entire function of $s$ satisfying the functional equation

$$
(2 \pi)^{-s} \Gamma(s) L_{F}(s)=(-1)^{\frac{1}{2} k}(2 \pi)^{s-k} \Gamma(k-s) L_{F}(k-s),
$$

where $\Gamma(s)$ denotes the gamma-function. In this paper we shall prove the following mean value result for $L_{F}$ on its critical line $\sigma=\frac{1}{2} k$.

Theorem. If $c_{-1}$ denotes the residue and $c_{-1} c_{0}$ the constant term in the Laurent expansion of

at $s=k$, then

$$
D(s)=\sum_{l=1}^{\infty}\left|a_{l}\right|^{2} l^{-s}
$$

$$
\int_{0}^{T}\left|L_{F}\left(\frac{1}{2} k+i t\right)\right|^{2} d t=2 c_{-1} T\left\{\log \frac{T}{2 \pi e}+c_{0}\right\}+O\left((T \log T)^{2 / 3}\right), \quad T \rightarrow \infty .
$$

This result enables us to make further progress towards the analogue of the Lindelöf hypothesis for the Dirichlet series under consideration. According to this hypothesis one expects

$$
L_{F}\left(\frac{1}{2} k+i t\right) \ll|t|^{\alpha}
$$

to hold for every $\alpha>0$. An approximate functional equation and

$$
\sum_{l \leqslant x}\left|a_{l}\right| \ll x^{\frac{1}{2}(k+1)}
$$

yield (2) with $\alpha=\frac{1}{2}$ (see [1, Corollary 3]). This improves the bound obtainable by the Phragmén-Lindelöf principle just slightly and may be regarded as the trivial 
estimate for (2). The first non-trivial estimate was proved in [2], where (2) was shown to hold for all $\alpha>5 / 12$. Our new mean value theorem now implies the

COROllary. On its critical line, $L_{F}$ satisfies the bound

$$
L_{F}\left(\frac{1}{2} k+i t\right) \ll|t|^{1 / 3}(\log |t|)^{5 / 6} .
$$

It should be noted that the corollary corresponds in a certain sense to the estimate

$$
\zeta\left(\frac{1}{2}+i t\right) \ll|t|^{1 / 6}(\log |t|)^{5 / 12}
$$

for the Riemann zeta-function $\zeta$. For there is some resemblance between $L_{F}\left(\frac{1}{2}(k-1)+s\right)$ and $\zeta^{2}(s)$. Notwithstanding great efforts, the exponent $1 / 6$ in (3) has only been improved by comparatively little in the last fifty years. Thus it is not very likely that a substantial improvement of our corollary will come from present-day methods.

The most straightforward ways to prove essentially (3) use estimates for exponential sums ( $c f$. [9, p. 85 and p. 92]). The non-trivial estimates for (2), however, are deduced from mean value theorems with good error terms. Up to a factor $|t|^{\varepsilon}$, $\varepsilon>0$, (3) can now also be obtained from a square mean result of $\zeta^{2}$. For Iwaniec [5] has shown that

$$
\int_{T}^{T+H}\left|\zeta\left(\frac{1}{2}+i t\right)\right|^{4} d t \ll H T^{\varepsilon}, \quad H=T^{2 / 3} .
$$

Our mean value theorem is in two ways of a more precise form than (4). We obtain an asymptotic result as well as a smaller error term.

At the very beginning of his proof Iwaniec employs the fact that the coefficients $d(l)$ in the Dirichlet series of $\zeta^{2}(s)$ are given by

$$
d(l)=\sum_{d \mid l} 1
$$

Since the arithmetic nature of our coefficients $a_{l}$ is still very mysterious and, in particular, no counterpart of (5) is known for them, Iwaniec's line of attack does not even get us started in the case of $L_{F}$. Here we further develop the method of [2] instead. It is not only improved but also considerably simplified.

Let us briefly outline the main steps of the proof. In $\$ 2$ we introduce the required facts of the spectral theory of the (hyperbolic) Laplacian on the modular curve and state two lemmas. In the first we summarize results already obtained in [2]. In the second we quote the main result of [3]. In $\$ 3$ we begin the proof of the theorem. We first proceed as in [2] starting from an approximate functional equation for $L_{F}$. Then some modifications are introduced mainly by the use of partial integrations. Here we further economize compared with [2]. Soon we can single out the part giving rise to the main term. The remainder term (15) is then shown to be the sum of a sum of residues and a contour integral. The appearance of these residues is closely connected with the discrete spectrum of the Laplacian on the modular curve. The functional equations in Lemma 1(iii) are used to split the contour integral into two parts. One of these parts is due to the presence of a continuous spectrum in the 
Laplacian on the modular curve. Together with the sum of residues it is majorized by Cauchy's inequality. This leaves us to bound a product of two double sums (cf. (33) to (35)). The first factor is estimated by Lemma 2, the second is treated in $\$ 4$. The part of the contour integral not yet dealt with is estimated in $\$ 5$. It turns out to be much smaller than our bound for the remainder term (15).

In $\$ 6$ we finally conclude the proof by working out the asymptotics of the main term and by deriving the Corollary.

In comparison with [2] we gain here at two places. The main result of [3] accounts for two thirds of our savings and $\$ 4$ for one third. The underlying reason for these savings is the use of summation formulae relating Fourier coefficients of non-holomorphic cusp forms with Kloosterman sums (cf. (38), (41)). There is a close affinity between them and Selberg's trace formula ( $c f$. [4]). In [2] we just used estimates for the square mean of those Fourier coefficients instead.

Thanks to Lemma 2 and $\S 4$ the contributions of the discrete and continuous spectrum to (15) can be handled simultaneously. This is a great simplification over [2]. It not only renders superfluous some awkward computations in [2], but also saves us from introducing certain auxiliary Dirichlet series. In [2] the latter were required to avoid the singularities of $(25)$ caused by the continuous spectrum in the half-plane $\sigma<\frac{1}{2}$.

Finally our new approach also clears the way for an extension of the results to cusp forms of other discrete groups. We can make the non-trivial remark that our method works for every finitely generated Fuchsian group of the first kind with cusps. The results in [3] have already been obtained in this generality. However, since we base our arguments partly on [2] where only the modular group was considered, we restrict ourselves to the latter again.

§2. Auxiliary lemmas. First we have to quote a number of results from the spectral theory of the Laplacian on the modular curve.

Let $\mathscr{H}$ denote the upper half-plane of complex numbers $z=x+i y, y>0$. Let $G=S L_{2}(\mathbb{R})$ act on $\mathscr{H}$ by linear fractional transformations.

Write $z_{M}=x_{M}+i y_{M}$ for

$$
z_{M}=\frac{a z+b}{c z+d}, \quad z \text { in } \mathscr{H} \text { and } M=\left(\begin{array}{ll}
a & b \\
c & d
\end{array}\right) \text { in } G .
$$

Let

and

$$
\Delta=y^{2}\left(\frac{\partial^{2}}{\partial x^{2}}+\frac{\partial^{2}}{\partial y^{2}}\right)
$$

$$
d \omega(z)=\frac{d x d y}{y^{2}}
$$

where $d x d y$ stands for the Lebesgue measure in the $z$-plane. The Laplacian $\Delta$ and the measure $d \omega$ are $G$-invariant. If $\mathbb{F}$ denotes a fundamental domain of $\Gamma=S L_{2}(\mathbb{Z})$ in $\mathscr{H}$, we define the scalar product $\langle$,$\rangle on \Gamma$-invariant functions $f$ and $g$ by

$$
\langle f, g\rangle=\int_{\mathfrak{z}} f(z) \bar{g}(z) d \omega(z)
$$


Next we choose a maximal system of $\Gamma$-invariant functions $\left(e_{j}\right)_{j=0}^{\infty}$ such that there are complex numbers $s_{j}$ with

$$
\Delta e_{j}+s_{j}\left(1-s_{j}\right) e_{j}=0
$$

and

$$
\left\langle e_{j}, e_{l}\right\rangle= \begin{cases}1, & \text { for } j=l \\ 0, & \text { otherwise }\end{cases}
$$

We may assume that $s_{0}=1$ and by a theorem of Roelcke $[8, \mathrm{p} .50]$ that $s_{j}=\frac{1}{2}+i t_{j}$ with $t_{j}>0$ for $j>0$. The functions $e_{j}, j \geqslant 0$, suffice to describe the contribution of the discrete spectrum to the spectral resolution of $\Delta$ on $\Gamma$-invariant functions. The portion coming from the continuous spectrum is governed by the Eisenstein series

$$
E(z, s)=\sum_{M \in \Gamma_{\infty} \backslash \Gamma} y_{M}^{s}, \quad \sigma>1
$$

where $\Gamma_{\infty}$ denotes the stabilizer of $\infty$ in $\Gamma$. Due to their $\Gamma_{\infty}$-invariance, these eigenfunctions of $\Delta$ enjoy Fourier expansions of the form

$$
\begin{aligned}
e_{0}(z) & =\left(\int_{\Im} d \omega(z)\right)^{-\frac{1}{2}}, \\
e_{j}(z) & =\sum_{l \neq 0} \alpha_{j l} y^{\frac{1}{2}} K_{i t_{j}}(2 \pi|l| y) e^{2 \pi i l x}, \quad j>0,
\end{aligned}
$$

and

$$
E(z, s)=y^{s}+\alpha_{0}(s) y^{1-s}+\sum_{l \neq 0} \alpha_{l}(s) y^{\frac{1}{2}} K_{s-\frac{1}{2}}(2 \pi|l| y) e^{2 \pi i l x}
$$

where the coefficients $\alpha_{j l}$ and $\alpha_{l}(s)$ are independent of $z$ and $K_{v}(z)$ is the modified Bessel function usually denoted in this way (cf. $[7$, p. 66]). It can be shown ( $c f .[6$, p. 46]) that

and for $l \neq 0$

$$
\alpha_{0}(s)=\pi^{\frac{1}{2}} \frac{\Gamma\left(s-\frac{1}{2}\right) \zeta(2 s-1)}{\Gamma(s) \zeta(2 s)}
$$

where

$$
\alpha_{l}(s)=2 \frac{|l|^{\frac{1}{2}-s} \sigma_{2 s-1}(l l \mid)}{\pi^{-s} \Gamma(s) \zeta(2 s)},
$$

$$
\sigma_{s}(l)=\sum_{\substack{d \mid l \\ d>0}} d^{s}
$$

In the notation just introduced the main results of [2, Section 5], are summarized in our next lemma.

LEMMA 1. Let $F$ be as in the introduction and

$$
f(z)=y^{k}|F(z)|^{2} .
$$


(i) If we define

$$
P_{0}(z, s, n)=\frac{(\pi n)^{s-\frac{1}{2}}}{\Gamma\left(s+\frac{1}{2}\right)} \sum_{M \in \Gamma_{x} \backslash \Gamma} y_{M}^{s} e^{2 \pi i n x_{M}}, \quad \sigma>1,
$$

for $n=1,2, \ldots$, we have

and

$$
\left\langle P_{0}(\cdot, s, n), f\right\rangle=(4 \pi)^{\frac{1}{2}-k} \frac{\Gamma(k+s-1)}{\Gamma\left(s+\frac{1}{2}\right)}\left(\frac{n}{4}\right)^{s-\frac{1}{2}} D(k+s-1, n)
$$

$$
\langle E(\cdot, s), f\rangle=(4 \pi)^{1-k-s} \Gamma(k+s-1) D(k+s-1,0),
$$

where, for $n=0,1, \ldots$,

$$
D(s, n)=\sum_{l=1}^{\infty} \frac{a_{l} \bar{a}_{l+n}}{\left(l+\frac{1}{2} n\right)^{s}}, \quad \sigma>k .
$$

(ii) The function $s \mapsto\langle E(\cdot, s), f\rangle$ is analytic in $\sigma \geqslant \frac{1}{2}$ except for a simple pole at $s=1$. The functions $s \mapsto\left\langle P_{0}(\cdot, s, n), f\right\rangle, n>0$, are analytic in $\sigma \geqslant \frac{1}{2}$, except for simple poles at $s=\frac{1}{2} \pm i t_{j}, j>0$, with residues

$$
\pm \frac{1}{2 i t_{j}} \sum_{s_{l}=s_{j}}\left\langle e_{l}, f\right\rangle \bar{\alpha}_{l n}
$$

(iii) For $n>0$ the functional equations

$\left\langle P_{0}(\cdot, s, n), f\right\rangle=\frac{\alpha_{n}(1-s)}{2 s-1}\langle E(\cdot, s), f\rangle+\left\langle P_{0}(\cdot, 1-s, n), f\right\rangle$

$$
+(4 \pi)^{\frac{1}{2}-k}\left(\frac{n}{4}\right)^{s-\frac{1}{2}} \frac{\Gamma(k+s-1)}{\Gamma\left(s+\frac{1}{2}\right)} \sum_{l=1}^{\infty} \frac{a_{l} \bar{a}_{l+n}}{\left(l+\frac{1}{2} n\right)^{k+s-1}} \Delta_{n}(s, l)
$$

hold, where

$$
\begin{aligned}
\Delta_{n}(s, l)=1+\frac{\Gamma(k-s) \Gamma\left(s-\frac{1}{2}\right)}{\Gamma(k+s-1) \Gamma\left(\frac{1}{2}-s\right)}\left(\frac{4 l+2 n}{n}\right)^{2 s+1} & \\
& -\pi^{\frac{1}{2}} \frac{\Gamma(k-s)}{\Gamma\left(\frac{1}{2}-s\right)}\left(\frac{4 l+2 n}{n}\right)^{s}\left(1-\left(\frac{n}{2 l+n}\right)^{2}\right)^{\frac{1}{2}(1-k)} \mathfrak{B}_{s-1}^{1-k}\left(\frac{2 l+n}{n}\right),
\end{aligned}
$$

and $\mathfrak{P}_{\nu}^{\mu}(z)$ is the Legendre function of the second kind usually denoted in this way (cf. [7, p. 152-153]).

Remark. Part (i) of Lemma 1 is nothing but (5.2) and (5.3) of [2]. Part (ii) follows from (5.4), (5.11) and (5.12) in [2]. Using Part (i), (3.8) in [2] and (6) we see that Part (iii) is equivalent to $[2,(5.13)]$. The estimates in $\$ 5$ below show that the infinite series in Part (iii) is absolutely and locally uniformly convergent for $-1<\sigma<2$.

The next lemma is our main result of [3] in the case when $\Gamma=S L_{2}(\mathbb{Z})$. 
LeMma 2. If $f$ is as in Lemma 1, then

$$
\sum_{0<t_{j} \leqslant T}\left|\left\langle f, e_{j}\right\rangle\right|^{2} e^{\pi t_{j}}+\frac{1}{4 \pi} \int_{-T}^{T}\left|\left\langle f, E\left(\cdot, \frac{1}{2}+i t\right)\right\rangle\right|^{2} e^{\pi|t|} d t \ll T^{2 k}
$$

§3. The main reduction. Let $\psi_{U}$ denote a $C^{\infty}$-function with support in the interval $[1-(1 / U), 2+(1 / U)], U \geqslant 2$, such that $\psi_{U}^{(j)}(\tau) \ll U^{j}$ for $j=0,1, \ldots$ and $\psi_{U}(\tau)=1$ for $1+(1 / U) \leqslant \tau \leqslant 2-(1 / U)$. Let

$$
I(T, U)=\int_{-\infty}^{\infty}\left|L_{F}\left(\frac{1}{2} k+i t\right)\right|^{2} \psi_{U}\left(\frac{t}{T}\right) d t
$$

By $[2,(2.15)$ to $(2.24)]$ we know that

$$
I(T, U)=\sum_{l, m=1}^{\infty} \frac{a_{l} \bar{a}_{m}}{(l m)^{\frac{1}{2} k}} G_{U}(l, m, T)+O(\log T), \quad 4 \leqslant U \leqslant T^{\frac{1}{2}},
$$

where

$$
G_{U}(l, m, T)=\int_{-\infty}^{\infty} \psi_{U}\left(\frac{t}{T}\right)\left\{\left(\frac{m}{l}\right)^{i t} \Phi\left(\frac{2 \pi l}{t}, \frac{2 \pi m}{t}\right)+\left(\frac{l}{m}\right)^{i t} \Phi^{*}\left(\frac{2 \pi l}{t}, \frac{2 \pi m}{t}\right)\right\} d t,
$$

$\Phi$ and $\Phi^{*}$ being real-valued $C^{\infty}$-functions of compact support. By [2, (2.19), (2.20)] these functions take the values $\Phi(\rho, \rho)=\phi(\rho), \Phi^{*}(\rho, \rho)=\phi^{*}(\rho)$ on the diagonal, where

$$
\phi^{*}(\rho)=1-\phi(1 / \rho)
$$

Here $\phi$ denotes the weight function occurring in our approximate functional equation [2, (2.5)]. As such it can be chosen with some freedom. Besides being an even real-valued $C^{\infty}$-function on the real numbers it has only to satisfy $(c f .[2,(2.4)])$

$$
\phi(\rho)=\left\{\begin{array}{cc}
1, & |\rho| \leqslant 2 / 3 \\
0, & |\rho| \geqslant 3 / 2
\end{array}\right\}
$$

Now let

$$
g_{n, U}(x)=\int_{-\infty}^{\infty} \psi_{U}(\tau)\left\{\phi\left(\frac{2 \pi x}{\tau}\right) e^{i \tau n / x}+\phi^{*}\left(\frac{2 \pi x}{\tau}\right) e^{-i \tau n / x}\right\} d \tau
$$

By the properties of $\psi_{U}, \phi, \phi^{*}, \Phi, \Phi^{*}$ listed above

$$
G_{U}(l, l, T)=T g_{0, U}(l / T),
$$

and, for positive $l, n$, the integrals $G_{U}(l, l+n, T), g_{n, U}\left(\left(l+\frac{1}{2} n\right) / T\right)$ vanish unless $l \ll T$ and $n \ll T$. Furthermore, since $\psi_{U}(\tau)=1$ for $1+(1 / U) \leqslant \tau \leqslant 2-(1 / U)$ integrations 
by parts yield, for $j=0,1, \ldots$,

and

$$
G_{U}(l, m, T) \ll\left|\log \frac{m}{l}\right|^{-j}\left(\frac{U}{T}\right)^{j-1}
$$

$$
T g_{n, U}\left(\frac{l+\frac{1}{2} n}{T}\right) \ll\left(\frac{l+\frac{1}{2} n}{n}\right)^{j}\left(\frac{U}{T}\right)^{j-1}
$$

Hence, for every $\varepsilon>0$,

$$
G_{U}(l, l+n, T) \ll \frac{1}{T}, \quad T g_{n, U}\left(\frac{l+\frac{1}{2} n}{T}\right) \ll \frac{1}{T},
$$

if $n U^{-1} T^{1-\varepsilon} \geqslant l \geqslant 1$ and $4 \leqslant U \leqslant T^{\frac{1}{2}}$. On the other hand, for $1 \leqslant n \leqslant U T^{\varepsilon}$, $n U^{-1} T^{1-\varepsilon} \leqslant l \ll T$ and $4 \leqslant U \leqslant T^{\frac{1}{2}}$ we have

$$
G_{U}(l, l+n, T)-T g_{n, U}\left(\frac{l+\frac{1}{2} n}{T}\right) \ll \frac{l}{n}\left[T\left(\frac{n}{l}\right)^{3}+\frac{n}{T}\right],
$$

if we integrate by parts just once and approximate the resulting integrand of $G_{U}(l, l+n, T)$ by that of

using

$$
T g_{n, U}\left(\frac{l+\frac{1}{2} n}{T}\right)
$$

$$
\begin{gathered}
\log (1+n / l)=\frac{n}{l+\frac{1}{2} n}+O\left(\left(\frac{n}{l}\right)^{3}\right), \\
\Phi\left(\frac{2 \pi l}{t}, \frac{2 \pi(l+n)}{t}\right)=\phi\left(\frac{2 \pi\left(l+\frac{1}{2} n\right)}{t}\right)+O\left(\frac{n}{|t|}\right), \\
\frac{d}{d t} \Phi\left(\frac{2 \pi l}{t}, \frac{2 \pi(l+n)}{t}\right)=\frac{d}{d t} \phi\left(\frac{2 \pi\left(l+\frac{1}{2} n\right)}{t}\right)+O\left(\frac{n}{t^{2}}\right),
\end{gathered}
$$

and similar estimates for $\Phi^{*}$ and $\phi^{*}$. Thus we obtain, from the well-known estimate

$$
\sum_{l \leqslant x}\left|a_{l}\right|^{2} \ll x^{k}
$$

and Cauchy's inequality,

$$
\begin{gathered}
\sum_{l, n=1}^{\infty} a_{l} \bar{a}_{l+n}\left\{(l(l+n))^{-\frac{1}{2} k} G_{U}(l, l+n, T)-T\left(l+\frac{1}{2} n\right)^{-k} g_{n, U}\left(\frac{l+\frac{1}{2} n}{T}\right)\right\} \\
\ll \sum_{l \leqslant n \leqslant U T^{\varepsilon} n T / U T^{\varepsilon} \leqslant l \ll T} \frac{\left|a_{l} a_{l+n}\right|}{\left(l+\frac{1}{2} n\right)^{k}}\left\{\frac{n}{l}+T\left(\frac{n}{l}\right)^{2}+\frac{l}{T}\right\}+O(1) \\
\ll \frac{\left(U T^{\varepsilon}\right)^{3}}{T}+U T^{\varepsilon} \ll U T^{3 \varepsilon},
\end{gathered}
$$


for $4 \leqslant U \leqslant T^{\frac{1}{2}}$ and every $\varepsilon>0$. Hence we conclude from (9) and (12) that for $4 \leqslant U \leqslant T^{\frac{1}{2}}$ and every $\varepsilon>0$

where

$$
I(T, U)=S_{0}(T, U)+2 \operatorname{Re}\left\{I_{1}(T, U)\right\}+O\left(U T^{e}\right),
$$

and for $n=0,1, \ldots$

$$
I_{1}(T, U)=\sum_{n=1}^{\infty} S_{n}(T, U)
$$

$$
S_{n}(T, U)=T \sum_{l=1}^{\infty} \frac{a_{l} \bar{a}_{l+n}}{\left(l+\frac{1}{2} n\right)^{k}} g_{n, U}\left(\frac{l+\frac{1}{2} n}{T}\right) .
$$

Mellin's inversion formula, (7) and (16) now yield for $n=0,1, \ldots$

$$
S_{n}(T, U)=\frac{1}{2 \pi i} \int_{(1)} K_{n, U}(s) D(k+s, n) T^{s+1} d s,
$$

where $\int_{(\rho)}$ stands for integration along the line $\sigma=\rho$ in the direction of increasing imaginary parts and

$$
K_{n, U}(s)=\int_{0}^{\infty} g_{n, U}(x) x^{s-1} d x, \quad \sigma>0 .
$$

By (6.1) to (6.5) of [2],

where

$$
K_{n, U}(s)=(2 \pi)^{-s} M_{n}(s+1) M(s+1, U),
$$

$$
\begin{gathered}
M_{n}(s)=\int_{-\infty}^{\infty} e^{2 \pi i n y} \tilde{\phi}(1 / y)|y|^{-s} d y, \quad \sigma>1, \\
\delta(x)= \begin{cases}\phi(x), & \text { if } x \geqslant 0, \\
\phi^{*}(x), & \text { if } x \leqslant 0,\end{cases}
\end{gathered}
$$

and

$$
M(s, U)=\int_{0}^{\infty} \psi_{U}(\tau) \tau^{s-1} d \tau .
$$

Our assumptions on $\psi_{U}$ imply that $M(\cdot, U)$ is an entire function and by $[2,(6.6)]$

$$
M(s, U) \ll \frac{1}{1+|s|}\left(\frac{U}{1+|s|}\right)^{c}
$$

holds for every $C \geqslant 1$ uniformly in $|\sigma| \leqslant 2$. If $n=1,2, \ldots$, we know from (6.10), (6.22) and (6.23) in [2] that $M_{n}$ is entire and satisfies

$$
M_{n}(s)=n^{s-1}\left|\frac{t}{2 \pi}\right|^{\frac{1}{-s}} e^{i t+i(\pi / 4) \operatorname{sgn} t}\left\{\delta\left(\frac{2 \pi n}{t}\right)+O\left(\frac{1+|t|}{(n+|t|)^{2}}\right)\right\}
$$

uniformly in $n \geqslant 1$ and $|\sigma| \leqslant 2$. 
Hence we obtain from (15), (17), (18) and Lemma 1(i)

$$
I_{1}(T, U)=\frac{1}{i} \int_{(2)} G(s, U) M(s, U)\left(\frac{T}{2 \pi}\right)^{s} d s
$$

with

$$
G(s, U)=(4 \pi)^{k-\frac{1}{2}} \frac{\Gamma\left(s+\frac{1}{2}\right)}{\Gamma(k+s-1)} \sum_{n=1}^{\infty}\left(\frac{4}{n}\right)^{s-\frac{1}{2}} M_{n}(s)\left\langle P_{0}(\cdot, s, n), f\right\rangle .
$$

Now let $\mathscr{C}$ denote a simple path from $\frac{1}{2}-i \infty$ to $\frac{1}{2}+i \infty$ in the half-plane $\sigma<\frac{1}{2}$ such that all singularities of $G(s, U)$ in $\sigma<\frac{1}{2}$ lie to the left of $\mathscr{C}$. By the method used to prove (7.12) in [2] it can be shown that there is a sequence of real numbers $\tau_{l} \rightarrow \infty$ for $l \rightarrow \infty$ such that the integrand of (24) tends to zero on $s=\sigma \pm i \tau_{l}, l \rightarrow \infty$, uniformly for $|\sigma| \leqslant 2$. Thus we have from (24)

$$
I_{1}(T, U)=\operatorname{Res}(T, U)+\frac{1}{i} \int_{\delta} G(s, U) M(s, U)\left(\frac{T}{2 \pi}\right)^{s} d s,
$$

where by Lemma $1($ ii) and (25) the contributions of the residues between $\mathscr{C}$ and the line $\sigma=2$ add up to

$$
\operatorname{Res}(T, U)=(4 \pi)^{k}\left(\frac{T}{8}\right)^{\frac{1}{2}} \sum_{j>0}\left\langle e_{j}, f\right\rangle \sum_{n=1}^{\infty} \bar{\alpha}_{j n}\left\{N_{n}\left(s_{j}, U\right)+N_{n}\left(1-s_{j}, U\right)\right\}
$$

with

$$
N_{n}(s, U)=\frac{\Gamma\left(s-\frac{1}{2}\right)}{\Gamma(k+s-1)} M_{n}(s) M(s, U)\left(\frac{2 T}{\pi n}\right)^{s-\frac{1}{2}} .
$$

Next we use the functional equations of Lemma 1(iii) to transform $G(s, U)$ on the right-hand side of (26). Then the integration over that part involving Eisenstein series is shifted back to the line $\sigma=\frac{1}{2}$. What remains is analytic in $-1<\sigma<\frac{1}{2}$. Thus we obtain from Lemma 1 and our assumptions on $\mathscr{C}$

where

$$
I_{1}(T, U)=\operatorname{Res}(T, U)+C(T, U)+R(T, U),
$$

$$
C(T, U)=(4 \pi)^{k}\left(\frac{T}{8}\right)^{\frac{1}{2}} \frac{1}{2 \pi i} \int_{\left(\frac{1}{2}\right)}\langle E(\cdot, s), f\rangle \sum_{n=1}^{\infty} \alpha_{n}(1-s) N_{n}(s, U) d s
$$

and

$$
R(T, U)=\frac{1}{i} \int_{(\varepsilon-1)} M(s, U)\left(\frac{T}{2 \pi}\right)^{s} \sum_{n=1}^{\infty} M_{n}(s) \sum_{l=1}^{\infty} \frac{a_{l} \bar{a}_{l+n} \Delta_{n}^{\prime}(s, l)}{\left(l+\frac{1}{2} n\right)^{k+s-1}} d s,
$$

with $0<\varepsilon<1$ and

$$
\Delta_{n}^{\prime}(s, l)=1-\pi^{\frac{1}{2}} \frac{\Gamma(k-s)}{\Gamma\left(\frac{1}{2}-s\right)}\left(\frac{4 l+2 n}{n}\right)^{s}\left(1-\left(\frac{n}{2 l+n}\right)^{2}\right)^{\frac{1}{2}(1-k)} \mathfrak{P}_{s-1}^{1-k}\left(\frac{2 l+n}{n}\right) .
$$

By Cauchy's inequality it follows from (22), (27), (28) and (30) that

$$
|\operatorname{Res}(T, U)+C(T, U)| \ll T^{\frac{1}{2}}\left(S_{1}(U) S_{2}(U)\right)^{\frac{1}{2}},
$$


where

$$
\begin{aligned}
S_{1}(U)= & \sum_{j>0}\left|\frac{\left\langle e_{j}, f\right\rangle}{t_{j}}\right|^{2} e^{\pi t_{j}}\left(1+t_{j}\right)^{(5 / 2)-2 k} \frac{U^{2}}{U^{2}+t_{j}^{2}} \\
& +\frac{1}{4 \pi} \int_{-\infty}^{\infty}\left|\frac{\left\langle E\left(\cdot, \frac{1}{2}+i t\right), f\right\rangle}{t}\right|^{2} e^{\pi|t|}(1+|t|)^{(5 / 2)-2 k} \frac{U^{2}}{U^{2}+t^{2}} d t
\end{aligned}
$$

and

$$
\begin{aligned}
S_{2}(U)= & \frac{1}{2} \sum_{j>0} e^{-\pi t_{j}}\left(1+t_{j}\right)^{-3 / 2} \frac{U^{2}}{U^{2}+t_{j}^{2}}\left(\left|\sum_{n=1}^{\infty} \frac{\bar{\alpha}_{j n} M_{n}\left(s_{j}\right)}{n^{i t_{j}}}\right|^{2}+\left|\sum_{n=1}^{\infty} \frac{\bar{\alpha}_{j n} M_{n}\left(1-s_{j}\right)}{n^{-i t_{j}}}\right|^{2}\right) \\
& +\frac{1}{4 \pi} \int_{-\infty}^{\infty} e^{-\pi|t|}(1+|t|)^{-3 / 2} \frac{U^{2}}{U^{2}+t^{2}}\left|\sum_{n=1}^{\infty} \alpha_{n}\left(\frac{1}{2}-i t\right) M_{n}\left(\frac{1}{2}+i t\right) n^{-i t}\right|^{2} d t
\end{aligned}
$$

Since for the modular group

$$
E\left(z, \frac{1}{2}\right)=0
$$

for all $z$ in $\mathscr{H}$ we conclude from Lemma 2 that

$$
S_{1}(U) \ll U^{\frac{1}{2}}
$$

$\S 4$. The estimate for $S_{2}(U)$. We define

$$
\begin{aligned}
S_{2}^{\prime}(U)= & \frac{1}{2} \sum_{j>0} h\left(s_{j}\right)\left(\left|\sum_{n=1}^{\infty} \frac{\bar{\alpha}_{j n} M_{n}\left(s_{j}\right)}{n^{i t_{j}}}\right|^{2}+\left|\sum_{n=1}^{\infty} \frac{\bar{\alpha}_{j n} M_{n}\left(1-s_{j}\right)}{n^{-i t_{j}}}\right|^{2}\right) \\
& +\frac{1}{4 \pi i} \int_{\left(\frac{1}{2}\right)} h(s)\left|\sum_{n=1}^{\infty} \alpha_{n}(1-s) M_{n}(s) n^{\frac{1}{2}-s}\right|^{2} d s
\end{aligned}
$$

with

$$
h(s)=\frac{p(s)}{\cos \pi s} \exp \left(\left(\frac{s-\frac{1}{2}}{U}\right)^{2}\right)
$$

In (37) $p$ denotes a polynomial of a sufficiently large degree $d$ in $s$ such that $h(s)=h(1-s)$ and $h\left(\frac{1}{2}(1+l)\right)=0$ for all integers $l$ with $|l| \leqslant \frac{1}{3} d-1$. Thus we obtain from (36)

$$
S_{2}^{\prime}(U)=\sum_{m, n=1}^{\infty}(m n)^{-\frac{1}{2}}\left\{\sum_{j>0} \alpha_{j m} \bar{\alpha}_{j n} \frac{g\left(s_{j}\right)+g\left(1-s_{j}\right)}{2}+\frac{1}{4 \pi i} \int_{\left(\frac{1}{2}\right)} \alpha_{m}(s) \bar{\alpha}_{n}(s) g(s) d s\right\},
$$

where

and

$$
g(s)=h(s) M_{m, n}(s)
$$

$$
M_{m, n}(s)=M_{n}(s) n^{1-s} M_{m}(1-s) m^{s}
$$


For the last equation we have had to assume, as we may by (10), (11) and (20), that $\mathcal{\phi}$ is an even function, whence by (19)

$$
\widetilde{M_{m}(s) m^{1-s}}=M_{m}(1-s) m^{s}
$$

on $\sigma=\frac{1}{2}$. This means that $g$ is an admissible weight function for the fundamental summation formulae expressing the curly brackets of (38) in terms of Kloosterman sums. More specifically, by Corollary 1 of [4], the term in those curly brackets equals

$$
\begin{aligned}
\frac{\delta_{m n}}{2 \pi^{2} i} \int_{\left(\frac{1}{2}\right)} g(s)(2 s-1) \cos \pi s d s & \\
& +\sum_{c=1}^{\infty} \frac{S(m, n, c)}{c \pi i} \int_{\left(\frac{1}{2}\right)}(g(s)+g(1-s))\left(s-\frac{1}{2}\right) J_{2 s-1}\left(\frac{4 \pi}{c}(m n)^{\frac{1}{2}}\right) d s,
\end{aligned}
$$

where $\delta_{m n}$ denotes the Kronecker symbol, $J_{v}$ the standard Bessel function of order $v$ (cf. $[7$, p. 65]) and $S(m, n, c)$ the Kloosterman sum

$$
\sum_{\substack{0<a<c \\(a, c)=1}} e^{2 \pi i(m a+n a-1) / c}
$$

with $a a^{-1} \equiv 1(\bmod c)$.

By (23) and (37) to (40), the first term of (41) contributes to the right-hand side of (38) at most

$$
\begin{aligned}
& \ll \sum_{n=1}^{\infty} \frac{1}{n} \int_{-\infty}^{\infty}|t|^{d+1} e^{-(t / U)^{2}}\left(\frac{|t|+1}{|t|+n}\right) d t \\
& \ll \int_{0}^{\infty} t^{d+1} e^{-(t / U)^{2}}\left(\sum_{n \leqslant t+1} \frac{1}{n}+\sum_{n \geqslant t+1} \frac{t+1}{n^{2}}\right) d t \ll U^{d+2} \log U .
\end{aligned}
$$

By Lemma 4 in [3], the contribution of the second term of (41) to the right-hand side of (38) equals

$$
\frac{1}{\pi} \sum_{m, n=1}^{\infty}(m n)^{-\frac{1}{2}} \sum_{c=1}^{\infty} \frac{S(m, n, c)}{c}\left\{\theta\left(\frac{2}{c}(m n)^{\frac{1}{2}}\right)+\theta\left(-\frac{2}{c}(m n)^{\frac{1}{2}}\right)\right\},
$$

where for $j=0,1, \ldots,\left[\frac{1}{3} d-1\right]$ the function $\theta$ admits the integral representation

with

$$
\theta(x)=(-2 \pi i x)^{j} \int_{-\infty}^{\infty} e^{2 \pi i x c h \rho} \hat{g}_{j}(2 \rho) d \rho
$$

$$
\hat{g}_{j}(2 \rho)=\frac{1}{2 \pi i} \int_{\left(\frac{1}{2}\right)} e^{(2 s-1) \rho} g_{j}(s) d s
$$


and the functions $g_{j}$ inductively given by

$$
\begin{aligned}
g_{0}(s) & =(g(s)+g(1-s))\left(s-\frac{1}{2}\right) \cos \pi s \\
& =p(s)\left(s-\frac{1}{2}\right) \exp \left(\left(\frac{s-\frac{1}{2}}{U}\right)^{2}\right) \cdot\left(M_{m, n}(s)+M_{m, n}(1-s)\right), \\
g_{j+1}(s) & =\frac{1}{4}\left\{\frac{g_{j}\left(s-\frac{1}{2}\right)}{s-1}-\frac{g_{j}\left(s+\frac{1}{2}\right)}{s}\right\}, \quad j \geqslant 0 .
\end{aligned}
$$

Thus we have by Cauchy's inequality and Plancherel's theorem

$$
\begin{aligned}
\theta(x) & \ll|x|^{j}\left(\int_{U|\rho| \leqslant 1}\left|\hat{g}_{j}(2 \rho)\right| d \rho+\int_{U|\rho| \geqslant 1}\left|\rho \hat{g}_{j}(2 \rho)\right| \frac{d \rho}{|\rho|}\right) \\
& \ll|x|^{j}\left\{\left(\frac{1}{U} \int_{-\infty}^{\infty}\left|\hat{g}_{j}(\rho)\right|^{2} d \rho\right)^{\frac{1}{2}}+\left(U \int_{-\infty}^{\infty}\left|\rho \hat{g}_{j}(\rho)\right|^{2} d \rho\right)^{\frac{1}{2}}\right\} \\
& \ll|x|^{j}\left\{\left(\frac{1}{U} \int_{-\infty}^{\infty}\left|g_{j}\left(\frac{1}{2}+i t\right)\right|^{2} d t\right)^{\frac{1}{2}}+\left(U \int_{-\infty}^{\infty}\left|\frac{d}{d t} g_{j}\left(\frac{1}{2}+i t\right)\right|^{2} d t\right)^{\frac{1}{2}}\right\} .
\end{aligned}
$$

It follows from (23), (37), (44) and (45) that

$$
g_{j}\left(\frac{1}{2}+i t\right) \ll(1+|t|)^{d+1-j} e^{-(t / U)^{2}}\left(\frac{|t|+1}{|t|+n}\right)^{2}\left(\frac{|t|+1}{|t|+m}\right)^{2},
$$

uniformly in $m, n \geqslant 1$. By Cauchy's theorem we deduce from (23) and (40) that

$$
\begin{aligned}
\frac{d}{d s} M_{m, n}(s)= & \frac{1}{2 \pi} \int_{0}^{2 \pi} M_{m, n}\left(s+e^{i v}\right) e^{-i v} d v \\
= & \frac{1}{2 \pi} \int_{0}^{2 \pi} \tilde{\phi}\left(\frac{2 \pi n}{t+\sin v}\right) \tilde{\phi}\left(-\frac{2 \pi m}{t+\sin v}\right) e^{-i v} d v \\
& +O\left(\frac{1}{1+|t|}\left(\frac{|t|+1}{|t|+n}\right)^{2}\left(\frac{|t|+1}{|t|+m}\right)^{2}\right)
\end{aligned}
$$

Since $\dot{\phi}(\tau)=0$ for $|\tau| \geqslant \frac{3}{2}$, the last integral vanishes if $|t| \leqslant 3$, whereas for $|t| \geqslant 3$

$$
\tilde{\phi}\left(\frac{2 \pi n}{t+\sin v}\right)=\tilde{\phi}\left(\frac{2 \pi n}{t}\right)+O\left(\frac{1}{1+|t|}\left(\frac{|t|+1}{|t|+n}\right)^{2}\right)
$$


Hence we obtain from (37), (44) and (45)

$$
\frac{d}{d t} g_{j}\left(\frac{1}{2}+i t\right) \ll(1+|t|)^{d+1-j}\left(\frac{1}{1+|t|}+\frac{1+|t|}{U^{2}}\right) e^{-(t / U)^{2}}\left(\frac{|t|+1}{|t|+n}\right)^{2}\left(\frac{|t|+1}{|t|+m}\right)^{2},
$$

uniformly in $m, n \geqslant 1$.

Thus we have by (46) to (48) for $0 \leqslant j \leqslant \frac{1}{3} d-1$

$$
\theta(x) \ll|x|^{j} U^{d+1-j}\left(\frac{U}{U+n}\right)^{2}\left(\frac{U}{U+m}\right)^{2}
$$

uniformly in $m, n, U \geqslant 1$ and all real $x$. Using this estimate with $j=0$ or $j=2$ respectively as well as the trivial bound

$$
|S(m, n, c)| \leqslant c
$$

we can therefore bound (43) by

$$
\begin{aligned}
& \ll U^{d+1} \sum_{m, n=1}^{\infty}(m n)^{-\frac{1}{2}}\left(\frac{U}{U+m}\right)^{2}\left(\frac{U}{U+n}\right)^{2}\left(\sum_{c \leqslant\left((m n)^{1 / 2}\right) / U} 1+\frac{m n}{U^{2}} \sum_{c \geqslant\left((m n)^{1 / 2}\right) / U} \frac{1}{c^{2}}\right) \\
& \ll U^{d}\left(\sum_{n \leqslant U} 1+U^{2} \sum_{n \geqslant U} \frac{1}{n^{2}}\right)^{2} \ll U^{d+2} .
\end{aligned}
$$

Together with (38) and (42) this yields

$$
S_{2}^{\prime}(U) \ll U^{d+2} \log U, \quad U \geqslant 2
$$

Thus integrating by parts we finally obtain from (34), (36) and (37)

$$
S_{2}(U) \ll U^{\frac{1}{2}} \log U
$$

Remark. A much better estimate could be obtained for (43) by analysing the functions $g_{j}$ more thoroughly. Such a more detailed analysis is, for example vital in the proof of Lemma 2 ( $c f$. Lemmas 5 to 7 in [3]). The reason why our crude estimate suffices here lies in the fact that for the inner sums in (36) the essential range of summation is $1 \leqslant n \ll U$.

§5. The estimate for $R(T, U)$. Using (22) and (23) we obtain from (31)

$$
R(T, U) \ll T^{\varepsilon-1} \frac{1}{i} \int_{(\varepsilon-1)}(1+|s|)^{\frac{1}{2}-\varepsilon}\left(\frac{U}{U+|s|}\right)^{4} \sum_{n=1}^{\infty} n^{\varepsilon-2}\left(\frac{|s|+1}{|s|+n}\right)^{2} \cdot\left|\sum_{l=1}^{\infty} \frac{a_{l} \vec{a}_{l+n} \Delta_{n}^{\prime}(s, l)}{\left(l+\frac{1}{2} n\right)^{k+s-1}}\right| d s .
$$

To estimate the sum over $l$ in $(50)$ we use several representations of $\mathfrak{P}_{v}^{\mu}(z)$ by the hypergeometric function $F(a, b ; c ; z)(c f .[7$, p. 37]). The formulae 1,10 and 13 on 
pages 155,157 and 158 of [7] say that for $x>1$

$$
\begin{aligned}
\mathfrak{P}_{s-1}^{1-k}(x) & \frac{1}{\Gamma(k)}\left(\frac{x-1}{x+1}\right)^{\frac{1}{2}(k-1)} F\left(1-s, s ; k ; \frac{1}{2}-\frac{1}{2} x\right) \\
= & \pi^{-\frac{1}{2}} \frac{\Gamma\left(\frac{1}{2}-s\right)}{\Gamma(k-s)}(2 x)^{-s}\left(1-x^{-2}\right)^{\frac{1}{2}(k-1)} F\left(\frac{1}{2}(k+s-1), \frac{1}{2}(k+s) ; s+\frac{1}{2} ; x^{-2}\right) \\
& +\pi^{-\frac{1}{2}} \frac{\Gamma\left(s-\frac{1}{2}\right)}{\Gamma(k+s-1)}(2 x)^{s-1}\left(1-x^{-2}\right)^{\frac{1}{2}(k-1)} F\left(\frac{1}{2}(k-s), \frac{1}{2}(k-s+1) ; \frac{3}{2}-s ; x^{-2}\right) \\
= & \frac{\left(x-\left(x^{2}-1\right)^{\frac{1}{2}}\right)^{s-\frac{1}{2}} \Gamma\left(\frac{1}{2}-s\right)}{(2 \pi)^{\frac{1}{2}}\left(x^{2}-1\right)^{\frac{1}{4}} \Gamma(k-s)} F\left(k-\frac{1}{2}, \frac{3}{2}-k ; s+\frac{1}{2} ; \frac{-x+\left(x^{2}-1\right)^{\frac{1}{2}}}{2\left(x^{2}-1\right)^{\frac{1}{2}}}\right) \\
& +\frac{\left(x-\left(x^{2}-1\right)^{\frac{1}{2}}\right)^{\frac{1}{2}-s} \Gamma\left(s-\frac{1}{2}\right)}{(2 \pi)^{\frac{1}{2}}\left(x^{2}-1\right)^{\frac{1}{4}} \Gamma(k+s-1)} F\left(k-\frac{1}{2}, \frac{3}{2}-k ; \frac{3}{2}-s ; \frac{-x+\left(x^{2}-1\right)^{\frac{1}{2}}}{2\left(x^{2}-1\right)^{\frac{1}{2}}}\right) .
\end{aligned}
$$

By (4.18) and (4.19) of [2] the first and the third equation in (51) yield

$$
\mathfrak{P}_{s-1}^{1-k}(x) \ll \begin{cases}(x-1)^{\frac{1}{2}(k-1)}, & \text { if } 1 \leqslant x \leqslant 1+(1+|s|)^{-2}, \\ \left(\mathrm{x}^{-\sigma}+x^{\sigma-1}\right) \frac{(1+|s|)^{\frac{1}{2}-k}}{\left(1-x^{-2}\right)^{\frac{1}{4}}}, & \text { if } x \geqslant 1+(1+|s|)^{-2},\end{cases}
$$

uniformly for $|\sigma| \leqslant 2$. Moreover by (32) and the second equation of (51) we have

$$
\begin{aligned}
& \Delta_{n}^{\prime}(s, l)=1-F\left(\frac{1}{2}(k+s-1), \frac{1}{2}(k+s) ; s+\frac{1}{2} ;\left(\frac{n}{2 l+n}\right)^{2}\right) \\
& \quad-\frac{\Gamma(k-s) \Gamma\left(s-\frac{1}{2}\right)}{\Gamma(k+s-1) \Gamma\left(\frac{1}{2}-s\right)}\left(\frac{4 l+n}{n}\right)^{2 s-1} F\left(\frac{1}{2}(k-s), \frac{1}{2}(k-s+1) ; \frac{3}{2}-s ;\left(\frac{n}{2 l+n}\right)^{2}\right) .
\end{aligned}
$$

Since by (4.13) and (4.14) of [2]

$$
F(a, b ; c ; z)=1+o\left(\left|\frac{a b z}{c}\right|\right)
$$

uniformly for all $a, b, c, z$ satisfying

$$
|z| \max _{l \geqslant 0}\left|\frac{(a+l)(b+l)}{(c+l)(l+1)}\right| \leqslant \frac{1}{2},
$$

we thus obtain from (32), (52) and (53), for sufficiently large $C$,

$$
\Delta_{n}^{\prime}(s, l) \ll \begin{cases}(1+|s|)\left(\frac{n}{l+n}\right)^{2}+\left(\frac{l+n}{n}\right)^{2 \sigma-1}, & \text { if } l \geqslant C(1+|s|)^{\frac{1}{2}} n, \\ 1+\left(\frac{l+n}{n}\right)^{2 \sigma-1}, & \text { if } n \leqslant l \leqslant C(1+|s|)^{\frac{1}{2}} n, \\ (n / l)^{\frac{1}{2}\left(k-\frac{1}{2}\right)}, & \text { if } 1 \leqslant l \leqslant n,\end{cases}
$$

uniformly in $l, n \geqslant 1$ and $|\sigma| \leqslant 2$. 
In (50) we now split the summation over $l$ into three parts according to the various estimates in (54). By (13) and Cauchy's inequality this leads to

$$
\begin{aligned}
\left|\sum_{l=1}^{\infty} \frac{a_{l} \bar{a}_{l+n}}{\left(l+\frac{1}{2} n\right)^{k+s-1}} \Delta_{n}^{\prime}(s, l)\right| \ll & n^{\frac{3}{2-\sigma-\frac{1}{2}} k^{\frac{1}{2}\left(k+\frac{1}{2}\right)}}+\left(n(1+|s|)^{\frac{1}{2}}\right)^{1-\sigma}+n^{1-2 \sigma} n^{\sigma} \\
& +(1+|s|) n^{2}\left((1+|s|)^{\frac{1}{2}} n\right)^{-\sigma-1} \ll n^{1-\sigma}(1+|s|)^{\frac{1}{2}(1-\sigma)},
\end{aligned}
$$

uniformly in $n \geqslant 1$ and $\varepsilon-1 \leqslant \sigma \leqslant-\varepsilon$ for positive $\varepsilon \leqslant \frac{1}{2}$. Inserting this bound into (50) we arrive at

$$
\begin{aligned}
R(T, U) & \ll T^{\varepsilon-1} \int_{-\infty}^{\infty}(1+|t|)^{\frac{3}{2}(1-\varepsilon)}\left(\frac{U}{U+|t|}\right)^{4} \sum_{n=1}^{\infty}\left(\frac{|t|+1}{|t|+n}\right)^{2} d t \\
& \ll T^{\varepsilon-1} \int_{0}^{\infty}(1+t)^{\frac{5}{2}-\frac{3}{2} \varepsilon}\left(\frac{U}{U+t}\right)^{4} d t \ll \frac{U^{7 / 2}}{T}\left(\frac{T}{U^{3 / 2}}\right)^{\varepsilon} .
\end{aligned}
$$

§6. The end of the proof. Next we treat $S_{0}(T, U)$, which gives rise to the main term. First we observe that by (6.7) and (6.8) of [2] $M_{0}$ is analytic except for a simple pole at $s=1$,

$$
M_{0}(s)=\frac{2}{s-1}+O(|s-1|), \quad s \rightarrow 1,
$$

and

$$
M_{0}(s) \ll(1+|s|)^{-2},
$$

uniformly for $|\sigma| \leqslant 2$. Hence we infer from (17), (18) and Lemma 1(i)

$$
\begin{aligned}
S_{0}(T, U)= & \frac{1}{i} \int_{(2)} M(s, U) M_{0}(s) D(k+s-1,0)\left(\frac{T}{2 \pi}\right)^{s} d s \\
= & T \operatorname{Res}_{s=1}\left\{M(s, U) M_{0}(s) D(k+s-1,0)\left(\frac{T}{2 \pi}\right)^{s-1}\right\} \\
& +\frac{1}{i} \int_{\left(\frac{1}{2}\right)} M(s, U) M_{0}(s) \frac{(4 \pi)^{k+s-1}}{\Gamma(k+s-1)}\langle E(\cdot, s), f\rangle d s .
\end{aligned}
$$

By (22), (57), Stirling's formula, Cauchy's inequality and Lemme 2 the last integral is at most

$$
\begin{aligned}
& \ll T^{\frac{1}{2}} \int_{-\infty}^{\infty}(1+|t|)^{-2-k}\left(\frac{U}{U+|t|}\right)^{2} e^{\frac{1}{2} \pi|t|}\left|\left\langle E\left(\cdot, \frac{1}{2}+i t\right), f\right\rangle\right| d t \\
& \ll T^{\frac{1}{2}}\left(\int_{-\infty}^{\infty}(1+|t|)^{-2-2 k}\left(\frac{U}{U+|t|}\right)^{2} e^{\frac{1}{2} \pi|t|}\left|\left\langle E\left(\cdot, \frac{1}{2}+i t\right), f\right\rangle\right|^{2} d t \int_{0}^{\infty}(1+t)^{-2}\left(\frac{U}{U+t}\right)^{2} d t\right)^{\frac{1}{2}}
\end{aligned}
$$

$\ll T^{\frac{1}{2}}$. 
By definition of $c_{-1}$ and $c_{0}$ we have

$$
D(k+s-1,0)=\frac{c_{-1}}{s-1}+c_{-1} c_{0}+O(|s-1|), \quad s \rightarrow 1
$$

whence we obtain from (56) and (58)

$$
S_{0}(T, U)=2 c_{-1} T\left(M(1, U)\left\{\log \frac{T}{2 \pi}+c_{0}\right\}+\left\{\frac{d}{d s} M(s, U)\right\}_{s=1}\right)+O\left(T^{\frac{1}{2}}\right) .
$$

Since by (21) and our assumptions on $\psi_{U}$

$$
M(1, U)=\int_{0}^{\infty} \psi_{U}(\tau) d \tau=\int_{1}^{2} d \tau+O(1 / U)=1+O(1 / U)
$$

and

$$
\left\{\frac{d}{d s} M(s, U)\right\}_{s=1}=\int_{0}^{\infty} \psi_{U}(\tau) \log \tau d \tau=\int_{1}^{2} \log \tau d \tau+O(1 / U)=2 \log 2-1+O(1 / U),
$$

we conclude that

$$
S_{0}(T, U)=2 c_{-1} T\left\{\log \frac{T}{2 \pi e}+c_{0}+2 \log 2\right\}+O\left(\frac{T}{U} \log T+T^{\frac{1}{2}}\right) .
$$

Thus we have for every $\varepsilon>0$ and $4 \leqslant U \leqslant T^{\frac{1}{2}}$

$$
\begin{aligned}
I(T, U)= & 2 c_{-1} T\left\{\log \frac{T}{2 \pi e}+c_{0}+2 \log 2\right\} \\
& +O\left(\frac{T}{U} \log T+(T U \log U)^{\frac{1}{2}}+\frac{U^{7 / 2}}{T} T^{\varepsilon}+U T^{\varepsilon}\right)
\end{aligned}
$$

in view of (14), (29), (33), (35), (49), (55) and (59). Since in (8) $\psi_{U}$ may be chosen either as a majorant or a minorant of the characteristic function of the interval $[1,2]$, we obtain from (60) by choosing $U=(T \log T)^{1 / 3}$ and $\varepsilon=\frac{1}{6}$

$$
\int_{T}^{2 T}\left|L_{F}\left(\frac{1}{2} k+i t\right)\right|^{2} d t=2 c_{-1} T\left\{\log \frac{T}{2 \pi e}+c_{0}+2 \log 2\right\}+O\left((T \log T)^{2 / 3}\right) .
$$

From (61) our theorem obviously follows by splitting the integration over $[1, T]$ into a sum of integrations over $\left[2^{-j-1} T, 2^{-j} T\right], 0 \leqslant j \leqslant \log T / \log 2$. 
It remains to prove the corollary. If $\sigma_{ \pm}= \pm 1 / \log t_{0}, t_{0} \geqslant 10$, we deduce from the functional equation (1) for $L_{F}$ and Stirling's formula

$$
\begin{aligned}
L_{F}^{2}\left(\frac{1}{2} k+i t_{0}\right) & =\frac{1}{2 \pi i} \int_{\left(\sigma_{+}\right)} L_{F}^{2}\left(\frac{1}{2} k+i t_{0}+s\right) \frac{e^{s^{2}}}{s} d s-\frac{1}{2 \pi i} \int_{\left(\sigma_{-}\right)} L_{F}^{2}\left(\frac{1}{2} k+i t_{0}+s\right) \frac{e^{s^{2}}}{s} d s \\
& \ll \int_{-\infty}^{\infty}\left|L_{F}\left(\frac{1}{2} k+\sigma_{+}+i\left(t_{0}+t\right)\right)\right|^{2}\left(1+\left|t_{0}+t\right|^{2 \sigma_{+}}\right) \frac{e^{-t^{2}}}{\left(t^{2}+\sigma_{+}^{2}\right)^{\frac{1}{2}}} d t .
\end{aligned}
$$

Similarly we have, for $\frac{1}{2} k<\sigma_{0}<\frac{1}{2}(k+1)$,

$$
\begin{aligned}
L_{F}^{2}\left(\sigma_{0}+i \tau_{0}\right) & =\frac{1}{2 \pi i} \int_{(1)} L_{F}^{2}\left(\sigma_{0}+i \tau_{0}+s\right) \frac{e^{s^{2}}}{s} d s-\frac{1}{2 \pi i} \int_{\left(\frac{1}{2} k-\sigma_{0}\right)} L_{F}^{2}\left(\sigma_{0}+i \tau_{0}+s\right) \frac{e^{s^{2}}}{s} d s \\
& \ll 1+\int_{-\infty}^{\infty}\left|L_{F}\left(\frac{1}{2} k+i\left(\tau_{0}+\tau\right)\right)\right|^{2} \frac{e^{-\tau^{2}}}{\left(\tau^{2}+\left(\frac{1}{2} k-\sigma_{0}\right)^{2}\right)^{\frac{1}{2}}} d \tau .
\end{aligned}
$$

Thus we obtain with $\sigma_{0}=\frac{1}{2} k+\sigma_{+}$and $\tau_{0}=t_{0}+t$

$$
\begin{aligned}
L_{F}^{2}\left(\frac{1}{2} k+i t_{0}\right) & \ll \int_{-\infty}^{\infty}\left(1+\left|t_{0}+t\right|^{2 \sigma_{+}}\right) \frac{e^{-t^{2}}}{\left(t^{2}+\sigma_{+}^{2}\right)^{\frac{1}{2}}} \\
& \times\left(1+\int_{-\infty}^{\infty}\left|L_{F}\left(\frac{1}{2} k+i\left(t_{0}+t+\tau\right)\right)\right|^{2} \frac{e^{-\tau^{2}}}{\left(\tau^{2}+\sigma_{+}^{2}\right)^{\frac{1}{2}}} d \tau\right) d t \\
& \ll \log \log t_{0}+\left(\log t_{0}\right) \int_{-\infty}^{\infty}\left|L_{F}\left(\frac{1}{2} k+i\left(t_{0}+t\right)\right)\right|^{2} e^{-\frac{1}{2} t^{2}} d t
\end{aligned}
$$

In the last integral an integration by parts makes our theorem applicable. It clearly implies

$$
L_{F}^{2}\left(\frac{1}{2} k+i t_{0}\right) \ll \log t_{0}\left(1+\int_{0}^{\infty} t e^{-\frac{1}{2^{2}}\{}\left\{\left(t_{0} \log t_{0}\right)^{2 / 3}+t \log t\right\} d t\right) \ll t_{0}^{2 / 3}\left(\log t_{0}\right)^{5 / 3}
$$

whence the collorary follows.

\section{References}

1. A. Good. Approximative Funktionalgleichungen und Mittelwertsätze für Dirichletreihen, die Spitzenformen assoziiert sind. Comm. Math. Helv., 50 (1975), 327-361.

2. A. Good. Beiträge zur Theorie der Dirichletreihen, die Spitzenformen zugeordnet sind. J. Number Theory, 13 (1981), 18-65. 
3. A. Good. Cusp forms and eigenfunctions of the Laplacian. Math. Ann., 225 (1981), 523-548.

4. A. Good. Local analysis of Selberg's trace formula. (To appear.)

5. H. Iwaniec. Fourier coefficients of cusp forms and the Riemann zeta-function. Bordeaux Sém. Theorie des Nombres, exp. no. 18. (1979/80), 1-36.

6. T. Kubota. Elementary theory of Eisenstein series (Kodansha, Tokyo, 1973).

7. W. Magnus, F. Oberhettinger and R. P. Soni. Formulas and theorems for the special functions of mathematical physics (Springer, Berlin, 3rd ed., 1966).

8. W. Roelcke. Ueber die Wellengleichung bei Grenzkreisgruppen erster Art. S.B. Heidelberger Akad. Wiss. Math. Nat. Kl. 4. Abh. (1956), 159-267.

9. E. C. Titchmarsh. The theory of the Riemann zeta-function (Oxford, 1951).

Dr. A. Good

Forschungsinstitut für Mathematik,

ETH-Zentrum

CH-8092 Zürich,

Switzerland.
10D12: NUMBER THEORY; Theory of modular forms; Modular forms, one variable.

Received on the 14th of September, 1981. 